\section{A PL1 program to perform multiserial correlation}

\author{
JAMES E. HAYS \\ The American University, Washington, D.C. 20016
}

and

\section{WILLIAM TAYLOR \\ Department of Medicine \\ George Washington University, Washington, D.C. 20005}

In many research situations in the behavioral sciences, it is desired to find the degree of relationship between two variables, one measured on an ordinal scale and the other measured at the interval level. Because the commonly used Pearson product moment correlation would be inappropriate in this situation, researchers commonly degrade the interval level variable to an ordinal level and apply one of the nonparametric rank order measures of relationship such as the Spearman rho or Kendall tau coefficients (Freeman, 1965). This procedure, although satisfactory for many applications, has the limitation that not all of the information contained in the interval level variable is utilized.

The multiserial correlation (Jaspen, 1946) is a procedure which enables one to find an accurate estimate of the degree of relationship between an ordinal level variable and an interval level variable. Since it utilizes all the information contained in the data, it is among the more powerful techniques available for this application. It requires the assumption that the ranks on the ordinal variable are based on an underlying normally distributed interval level scale.

If this assumption is made, it is possible to convert from percentile ranks to $\mathrm{z}$ (standard) scores through an inverse normal probability function. For this procedure to be valid, it is necessary to assume that each $S$ in a given category, $k$, of the ordinal variable is at the mean of that category on the corresponding underlying interval scale. Consequently, the mean $z$ value of the underlying scale for that category is given by the formula:

$$
\overline{\mathrm{z}}_{\mathrm{k}}=\frac{\mathrm{Y}_{\mathrm{L}_{\mathrm{k}}}-\mathrm{Y}_{\mathrm{U}_{\mathrm{k}}}}{\mathrm{p}_{\mathrm{k}}},
$$

where the $\mathrm{Y}_{\mathrm{L}_{\mathrm{k}}}$ and $\mathrm{Y}_{\mathrm{U}_{\mathrm{k}}}$ are the lower and upper ordinates of the segment of the standard normal distribution corresponding to the ordinal category, $k$, and where $p_{k}$ is the proportion of cases in that category. Using these values of $\bar{z}_{k}$ and the original values of the interval level variable, one may apply the standard Pearson procedure to obtain the multiserial correlation. The resulting coefficient is useful when generalization is to be made to the same set of ordinal categories that were employed in the collection of the ordinal level data.
It is also possible to estimate, by means of the appropriate transformation, the correlation between the interval level variable and the continuum hy pothesized to underlie the ordinal variable. This transformation is essentially a correction for grouping. As a result of grouping the underlying interval level scale values into ordinal categories, the multiserial coefficient obtained from the grouped data will be smaller than the corresponding Pearson coefficient which would have been obtained if interval level data had been available for both variables. The appropriate transformation to obtain a corrected multiserial correlation is to divide the obtained coefficient by the assumed correlation between the underlying interval variable and the midpoints of the ordinal categories. This assumed correlation, $\mathrm{r}_{\mathrm{z}} \overline{\mathrm{z}}$ is equal to the standard deviation of the $\overline{\mathrm{z}}$ scores corresponding to the midpoints of the ordinal categories. The formula for the corrected multiserial is therefore:

$$
\mathrm{cr}_{\mathrm{ms}}=\frac{\mathrm{r}_{\mathrm{ms}}}{\mathrm{s}_{\overline{\mathbf{z}}}},
$$

where $s_{\overline{\mathrm{Z}}}$ is the standard deviation of the $\overline{\mathrm{z}}$ values used in the calculation of the multiserial coefficient.

Since the multiserial correlation is a form of the Pearson coefficient, the same quantity,

$$
\frac{\mathrm{r}_{\mathrm{ms}} \sqrt{\mathrm{N}-2}}{\sqrt{1-\mathrm{r}_{\mathrm{ms}}^{2}}}
$$

can be used to test the significance of the obtained $r_{m s}$. The value resulting from the application of this equation is distributed as $t$ with $\mathrm{N}-2$ degrees of freedom. This statistic can be used to test either the nondirectional hypothesis, $\mathrm{H}_{\mathrm{o}}: \mathrm{r}_{\mathbf{m s}}=$ 0 , or the directional hypothesis, $\mathrm{H}_{\mathrm{o}}: \mathrm{r}_{\mathbf{m s}} \geqslant 0$ or $\mathrm{H}_{\mathrm{o}}: \mathrm{r}_{\mathbf{m s}} \leqslant 0$.

Input. Data are read according to a user-supplied format in pairs $\left(Y_{i}, X_{i}\right)$ where $Y_{i}$ is the ordinal level observation for Subject $\mathrm{i}$ and $\mathrm{X}_{\mathrm{i}}$ is the corresponding interval level observation.

Output. Output consists of the following: (1) a summary of the data, (2) the value of the multiserial correlation, (3) the corrected multiserial correlation, (4) the value of the test statistic, $t$, and (5) the corresponding degrees of freedom.

Computer and Language. The program was written in PL1 and has been tested on a IBM 370/145.

Availability. Source decks, test data, sample output, and instructions for use can be obtained free of charge from James $\mathrm{E}$. Hays, Department of Psychology, The American University, Washing ton, D.C. 20016.

\section{REFERENCES}

Freeman, L. C. Elementary applied statistics for students in behavioral science. New York: Wiley, 1965.

Jaspen, N. Serial correlation. Psychometrika, 1946, 11, 23-30. 